\section{Using ferruginous lake waters as source of Fe-metabolizing electroactive bacteria to produce biomodified electrodes and induce $\mathrm{Fe}$ - bearing nano-particles precipitation}

ASTOLFO VALERO, MSC. ${ }^{1,2}$, KARELYS UMBRÍASALINAS $^{3}$, JIŘÍ JAN ${ }^{3}$, JAKUB BOROVEC ${ }^{3}$ AND DANIEL A PETRASH ${ }^{3,4}$

${ }^{1}$ Biology Centre CAS - SoWa

${ }^{2}$ University of South Bohemia

${ }^{3}$ Biology Centre CAS

${ }^{4}$ Czech Geological Survey

Presenting Author: astolfo.valero@bc.cas.cz

The production of electric energy by microbes that transfer electrons to insoluble materials had enabled the development of microbial electrochemical technologies. These are increasingly applied to wastewater treatment and bioremediation. Nevertheless, the applicability of biomodified electrodes to study biogeochemical transformations of Fe-oxyhydroxides is yet to be further explored. For this purpose, we are using the genomic potential of environmental samples retrieved from an engineered redox stratified lake (Lake Medard, NW Czechia) that displays a $\mathrm{Fe}(\mathrm{II})$-rich anoxic monimolimnion. There, the presence of electroactive Fe-metabolizing bacteria (e.g., Geobacter sp., Rhodoferrax sp.) has been reported[1]. Our aim is to review the efficiency of biomodified electrodes produced by using bottom water samples from this lake. Accordingly, we submerged graphite rod electrodes inside an electrolyte solution $(n=3)$ containing environmental sample and a growth medium (sodium acetate $10 \mathrm{mM}$ as carbon source). Afterwards, we applied an $E=$ $+0.2 \mathrm{~V}$ ( $v s$. $\mathrm{Ag} / \mathrm{AgCl}$ ) during $\sim 25$ days[2]. This allowed us to obtain a biomodified electrode capable of producing current densities up to $10 \mathrm{~mA} \mathrm{~cm}^{-2}$ (Figure 1a). These are, however, 25x lower than control experiments with Geobacter sp.-coated electrodes (i.e., maximum $j=250 \mathrm{~mA} \mathrm{~cm}{ }^{-2}$; Figure 1b). Consequently, we expect that our biofilms are composed by a diverse anaerobes' consortium rather than solely model Geobacter species. Identification of the electroactive bacteria composing our biofilms by $16 \mathrm{~s}$ rRNA gene is underway. We have also collected particles precipitated in equilibrium with our experimental solutions and characterized them by in situ spectroscopy. The Lake Medard' Fe-metabolizing biofilms coating the electrode surface appear to stabilize Fe(III)-bearing particles by oxidizing acetate while utilizing the electrode, not iron as a terminal electron acceptor. This would open the possibility of utilizing these biomodified graphite electrodes to induce Fe-oxyhydroxide mineral transformations in batch experiments, take advantage of their affinity for critical metals, such as REE, or prevent the internal release of P. Therefore, we are performing biomineralization experiments after introducing our biomodified electrodes in amended dissolved and particulate Fe-rich solutions.

[1] Petrash et al. Env. Sci. Process. Impacts 20, 1414-1426
[2] Fricke et al. Energy. Environ. Sci. 1, 144-147 (2008).
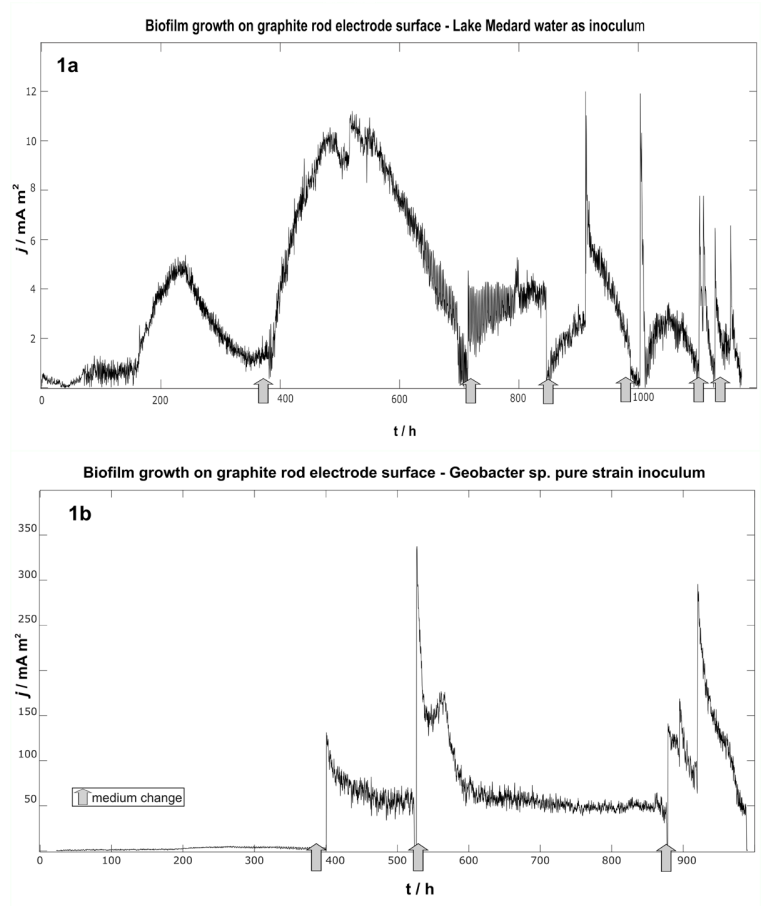